\title{
Effects of changes in smoking status on risk estimates for myocardial infarction among women recruited for the Royal College of General Practitioners' Oral Contraception Study in the UK
}

\author{
Vicci Owen-Smith, Philip C Hannaford, Maria Warskyj, Susan Ferry, Clifford R Kay
}

\begin{abstract}
Study objective-To determine whether changes in smoking status among women recruited for the Royal College of General Practitioners' Oral Contraception Study affected previous risk estimates for myocardial infarction.

Design-(1)Postal survey between November 1994 and July 1995 of women still under general practitioner observation. Validation of the smoking information supplied by the women on the questionnaire by comparison with that reported by the general practitioner at recruitment to the main study. (2) Nested case-control study of 103 cases of myocardial infarction, matched with 309 controls, to see if different risk estimates were obtained when smoking status at recruitment or smoking status at time of event were used in the analysis.
\end{abstract}

Setting- 650 general practices throughout the United Kingdom.

Participants-10 073 women who responded to the questionnaire $(85.4 \%$ of 11797 sent out).

Main results-There was good agreement between smoking information recorded by the general practitioner at recruitment and that supplied retrospectively by respondents to the questionnaire. The risk estimates for myocardial infarction associated with use of combined oral contraceptives (COCs) were almost identical irrespective of whether smoking status at recruitment or at time of event was used for the statistical adjustment. This was because few women stopped smoking while also using COCs. In fact, fewer regular smokers who have ever used COCs reported stopping smoking than never users. The risk estimates for myocardial infarction associated with smoking were smaller when smoking habits at recruitment was used than when smoking habits at time of event was used.

Conclusions-Previous results from the Oral Contraception Study regarding the effects of COCs are unlikely to have been biased by changes in the smoking habits of the cohort, but the effects of smoking have probably been underestimated. There is still a need for effective health education regarding the risks associated with smoking, particularly among users of COCs.

(F Epidemiol Community Health 1998;52:420-424)

Since its inception in 1968 the Royal College of General Practitioners' (RCGP) Oral Contraception Study has made a major contribution to our understanding of the health effects of combined oral contraceptives (COCs). For example, the study was among the first to show that the risk of dying from arterial disease is increased in older users, and users who smoke. ${ }^{1}$ In later years the study also found increased risks of myocardial infarction, ${ }^{2}$ and fatal stroke ${ }^{3}$ in COC users who smoke. These findings continue to have an important influence on clinical practice. We have been aware, however, that these analyses were based on the cohort's smoking habits recorded at recruitment to the study. Bias may have occurred if there was a material difference in the proportion of COC users and non-users who changed their smoking status since recruitment. In late 1994/early 1995 we obtained from a postal survey of all women remaining under general practitioner observation, up to date information about smoking. Using this information we have conducted a nested case-control study of myocardial infarction to determine whether such bias had been introduced.

\section{Methods}

RCGP ORAL CONTRACEPTION STUDY

During a 14 month period starting in May 1968, 1400 general practitioners throughout the United Kingdom interviewed and recruited 23000 women who were using oral contraceptives and a similar number of women who had never done so. ${ }^{4}$ The two groups were of similar age (average 29 years), all were married or living as married, most were white. Information collected at recruitment included husband's occupation (for determining social class), parity, previous use of COCs, significant past medical history, and number of cigarettes currently smoked each day (ex-smokers were not distinguished from non-smokers). Since recruitment, the general practitioners have supplied for all subjects still under their care, information about use of hormonal preparations, all newly presenting episodes of illness, surgery, pregnancy and, when appropriate, date and cause of death. 
Table 1 Comparison between information about smoking habits at recruitment to the Oral Contraception Study as supplied by the general practitioner compared with that supplied by the women in 1994/95 (\% of total)

Retrospective estimate in Daily cigarette consumption at recruitment recorded by general practitioner 1994/95 of daily cigarette in 1968/69

consumption at

\begin{tabular}{lllll}
$\begin{array}{l}\text { rocruitment } \\
\text { rensumption }\end{array}$ & Nil & $1-14$ & $15+$ & Total \\
\hline Nil & $5482(56.9)$ & $470(4.9)$ & $42(0.4)$ & $5994(62.2)$ \\
$1-14$ & $166(1.7)$ & $996(10.3)$ & $244(2.5)$ & $1406(14.6)$ \\
$15+$ & $158(1.6)$ & $926(9.6)$ & $1147(11.9)$ & $2231(23.2)$ \\
Total & $5806(60.3)$ & $2392(24.8)$ & $1433(14.9)$ & 9631 \\
\hline
\end{tabular}

POSTAL QUESTIONNAIRE

Between November 1994 and July 1995, the practices of the 12303 women (26.5\% of the original cohort) still under general practitioner observation were asked to forward health survey questionnaires on our behalf. Most agreed to do so, dispatching questionnaires to 11797 women. The questionnaires were sent via the participating practices because the research unit did not hold identifying information about the women; all correspondence between the unit and participating doctors about individual subjects uses a unique study number, the key to which is held only by the family doctor. This system ensures patient confidentiality. The recipient was asked to complete the questionnaire before returning it directly to the research unit. If the questionnaire was not returned, second and third mailings were sent, again via the general practitioner. By comparing information about the date of birth, pregnancies, and history of hysterectomy given on the returned forms with records already held in the unit, we were able to validate that the general practitioners had sent the questionnaire to the correct woman.

The questionnaire asked about a number of lifestyle issues, including lifetime smoking habits. In the questionnaire a regular smoker was defined as someone who has smoked at least one cigarette a day for more than one year. Using this definition, each woman was asked whether she had ever smoked regularly and if so, her age at starting, number of cigarettes currently smoked and, if applicable, age and daily cigarette consumption at stopping. From this information we were able to estimate whether each woman was a smoker or nonsmoker at recruitment and, if included in the nested case-control study, her smoking status at time of the event.

NESTED CASE-CONTROL STUDY OF MYOCARDIAL INFARCTION

By July 1996, 238 women had had a first ever episode of myocardial infarction (International Classification of Diseases (ICD), 8th revision code 410 ) reported to the study; 103 (43.2\%) of these events occurred in women who had completed the postal questionnaire. These women were used to assess whether changes in smoking status during study follow up had biased the estimated risk of myocardial infarction associated with COC use. Each case (index subject) was matched with three controls who had completed the postal questionnaire and who were: born within six months of the index subject's date of birth; still under observation when infarction was diagnosed in the index subject; had no history of myocardial infarction before the date of the index subject's diagnosis; were recruited by a different general practitioner from that of the case. Within each matched set, information about exposure status related to that which pertained on the date of the index subject's myocardial infarction. Data included in the analysis were: social class at recruitment, smoking status at recruitment (as supplied by the general practitioner on the recruitment form), smoking status at time of event (as determined from the information supplied by the woman on the postal questionnaire), COC status at time of event (never, current, former), history of hypertension (ICD 401) or toxaemia of pregnancy (ICD 6370, 6371 and 6379) at time of event. As we only had age in full years for women who stopped smoking, we were unable to tell whether the 26 cases and two controls who reported stopping at the same age as their corresponding index subject's infarction did so before or after the event. These women, therefore, were classified as current smokers on the assumption that the effects of smoking are likely to take a number of months, if not years, to disappear. Women whose age at stopping was younger than the age at the time of event were classified as ex-smokers. The data were examined using the conditional logistic regression program available in EGRET. ${ }^{5}$ Two sets of adjusted odds ratios were obtained. The first were adjusted for social class, history of hypertension, and smoking status at recruitment. The second were adjusted for social class, history of hypertension and smoking status at time of event.

\section{Results}

Of the 11797 questionnaires sent out by the general practitioners, 10073 (85.4\%) were returned. A few questionnaires could not be used for this report because: the woman's smoking status at recruitment was unknown (27 women); all or parts of the questionnaire were incomplete including the key question about ever smoked regularly (120); there were missing or inconsistent responses to the remaining questions about smoking (199); the stated age at starting (47) or stopping smoking (49) was the same as that for recruitment (this information could not be used as we could not determine accurately the woman's smoking status at month of recruitment). We were thus left with complete smoking data for 9631 women $(95.6 \%$ of those who returned questionnaires).

The level of agreement between information supplied by the general practitioners at recruitment to the study and that reported by the women in the questionnaire was good (table 1). This indicated that it was reasonable to use the health survey data in this report.

Fewer regular smokers who had ever used COCs by 1993 reported stopping smoking than regular smokers who had never used COCs (table 2). The mean (SD) age at stopping smoking was 41.5 (11.7) years; median age 41.0 years. This suggests that most 
Table 2 Number and percentage of women who reported ever smoking regularly and who stated that they had stopped, by personal characteristics (95\% confidence intervals)

\begin{tabular}{|c|c|c|c|}
\hline & $\begin{array}{l}\text { Number } \\
\text { stopping/all women }\end{array}$ & $\%$ & $(95 \% C I)$ \\
\hline \multicolumn{4}{|l|}{ Ever used COCs to 1993} \\
\hline Never & $803 / 1362$ & 59.0 & (56.3 to 61.6$)$ \\
\hline Ever & $1462 / 2912$ & 50.2 & (48.4 to 52.0$)$ \\
\hline \multicolumn{4}{|l|}{ COC status at recruitment } \\
\hline Never & $1160 / 2117$ & 54.8 & (52.7 to 56.9$)$ \\
\hline Current & $1105 / 2157$ & 51.2 & (49.1 to 53.3$)$ \\
\hline \multicolumn{4}{|l|}{ Age at recruitment } \\
\hline$<20$ & $94 / 243$ & 38.7 & (32.6 to 44.8 ) \\
\hline $20-24$ & $448 / 926$ & 48.4 & (45.2 to 51.6$)$ \\
\hline $25-29$ & $576 / 1145$ & 50.3 & (47.4 to 53.2 ) \\
\hline $30-34$ & $526 / 952$ & 55.3 & (52.1 to 58.4$)$ \\
\hline $35+$ & $621 / 1008$ & 61.6 & (58.6 to 64.6$)$ \\
\hline \multicolumn{4}{|l|}{ Social class at recruitment } \\
\hline Non-manual & $738 / 1153$ & 64.0 & (61.2 to 66.8$)$ \\
\hline Manual & $1524 / 3108$ & 49.0 & ( 47.3 to 50.8 ) \\
\hline Unknown & $3 / 13$ & 23.1 & $(1.9$ to 45.5$)$ \\
\hline \multicolumn{4}{|l|}{ Place of residence at recruitment } \\
\hline Scotland & $411 / 862$ & 47.7 & (44.3 to 51.0$)$ \\
\hline Northern England/Midlands & $966 / 1847$ & 52.3 & (50.0 to 54.6$)$ \\
\hline Southern England & $785 / 1373$ & 57.2 & ( 54.6 to 59.8 ) \\
\hline Wales & $100 / 187$ & 53.5 & ( 46.3 to 60.6$)$ \\
\hline $\mathrm{N}$ Ireland & $3 / 5$ & 60.0 & (14.7 to 94.7$)$ \\
\hline \multicolumn{4}{|l|}{ Parity at recruitment } \\
\hline 0 & $310 / 553$ & 56.1 & (51.9 to 60.2 ) \\
\hline $1-3$ & $1716 / 3233$ & 53.1 & ( 51.4 to 54.8 ) \\
\hline $4+$ & $239 / 488$ & 49.0 & (44.5 to 53.5$)$ \\
\hline \multicolumn{4}{|c|}{ Daily cigarette consumption at recruitment, as reported by the general practitioner ${ }^{\star}$} \\
\hline $1-14$ & $1140 / 2086$ & 54.7 & (52.5 to 56.8$)$ \\
\hline $15+$ & $526 / 1415$ & 37.2 & (34.7 to 39.7$)$ \\
\hline \multicolumn{4}{|c|}{ Estimated body mass index at age $30 t$} \\
\hline$<20$ & $312 / 655$ & 47.6 & (43.8 to 51.5$)$ \\
\hline $20-24$ & $1471 / 2710$ & 54.3 & ( 52.4 to 56.2 ) \\
\hline $25+$ & $441 / 828$ & 53.3 & (49.9 to 56.7 ) \\
\hline
\end{tabular}

*Excludes 324 women whom the general practitioner recorded as being a non-smoker even though the woman reported being a smoker, 291 women who stopped smoking before recruitment, and 158 women who started after recruitment. + Calculated from self reported weight at age 30 and reported height at time of completing the questionnaire.

Table 3 Association of first ever myocardial infarction with smoking habits, use of COCs, history of hypertension, and social class

\begin{tabular}{|c|c|c|c|c|}
\hline & $\begin{array}{l}\text { Number of } \\
\text { cases: } \\
\text { controls }\end{array}$ & $\begin{array}{l}\text { Unadjusted OR } \\
(95 \% \mathrm{CI})\end{array}$ & $\begin{array}{l}\text { Adjusted } O R^{\star} \\
(95 \% \mathrm{CI})\end{array}$ & $\begin{array}{l}\text { Adjusted ORt } \\
(95 \% \mathrm{CI})\end{array}$ \\
\hline \multicolumn{5}{|c|}{ Smoking status at recruitment using general practitioner supplied information } \\
\hline Non-smoker & $36: 205$ & 1.0 & 1.0 & \\
\hline Smoker & $67: 104$ & $3.4(2.2$ to 5.5$)$ & $3.6(2.2$ to 5.9$)$ & \\
\hline \multicolumn{5}{|c|}{ Smoking status at event using health survey information } \\
\hline Non-smoker & $44: 241$ & 1.0 & & 1.0 \\
\hline Smoker & $59: 68$ & $4.8(2.9$ to 7.8$)$ & & $5.1(3.0$ to 8.7$)$ \\
\hline \multicolumn{5}{|c|}{ COC status at event } \\
\hline Never user & $38: 156$ & 1.0 & 1.0 & 1.0 \\
\hline Current user & $8: 13$ & $2.9(1.0$ to 8.7$)$ & $2.9(0.9$ to 9.6$)$ & $2.7(0.8$ to 9.0$)$ \\
\hline Former user & $57: 140$ & $1.7(1.0$ to 2.8$)$ & $1.6(1.0$ to 2.8$)$ & $1.6(1.0$ to 2.8$)$ \\
\hline \multicolumn{5}{|c|}{ History of hypertension at event } \\
\hline No & $69: 252$ & 1.0 & 1.0 & 1.0 \\
\hline Yes & $34: 57$ & $2.3(1.4$ to 4.0$)$ & $2.6(1.5$ to 4.8$)$ & $2.9(1.6$ to 5.3$)$ \\
\hline \multicolumn{5}{|c|}{ Social class at recruitment } \\
\hline Non-manual & $73: 195$ & 1.0 & 1.0 & 1.0 \\
\hline Manual & $30: 114$ & $0.7(0.4$ to 1.1$)$ & $0.9(0.6$ to 1.6$)$ & $0.9(0.5$ to 1.5$)$ \\
\hline
\end{tabular}

${ }^{\star}$ Odds ratio adjusted for social class and smoking status at recruitment, history of hypertension at event, except where variable itself is being examined. †Odds ratio adjusted for social class at recruitment, smoking status and history of hypertension at event, except where variable itself is being examined. Additional adjustment for toxaemia of pregnancy was not possible as only two cases and 0 controls had such a history recorded.

women stopped smoking at an age when they would already have stopped using COCs. This was confirmed by examining the smoking habits of women in the case-control study; only one of the cases and none of the controls who were current users at the time of the event had changed from being a smoker at recruitment to non-smoker at myocardial infarction. As a consequence, the risk estimates for myocardial infarction associated with COC use were almost identical irrespective of which smoking variable was used for the adjustment (table 3).

The risk of myocardial infarction associated with smoking was smaller when cigarette consumption at recruitment was used (adjusted odds ratio (OR) 3.6, 95\% CI 2.2 to 5.9 ) than when smoking status at time of event was used (adjusted OR 5.1, 95\% CI 3.0 to 8.7; table 3). This finding was as expected as we have previously argued ${ }^{2}$ that it is probable that many women will stop smoking during the study and few start, resulting in an underestimation of the effects of smoking when information collected at recruitment is used. Indeed, according to the questionnaire data, 4274 women had ever smoked regularly, of whom $2265(53.0 \%)$ have stopped, whereas only $267(4.5 \%)$ of the 5994 non-smokers at recruitment subsequently started.

The mean (SD) number of years smoked among the ex-smokers was 22.5 (11.7) years. More of the older women at recruitment had stopped smoking than younger recruits (table 2). In addition, more women of higher social class, living in southern England, of nulliparity, and with a lower daily cigarette consumption at recruitment stopped smoking than those without these characteristics. There was no consistent pattern of stopping smoking with estimated body mass index at age 30 .

Most women who stopped smoking indicated that they did so for personal reasons $(83.1 \%)$, followed by doctors advice $(8.7 \%)$, pregnancy $(8.0 \%)$, and illness $(7.9 \%)$ (the percentages total more than $100 \%$ because some women gave more than one reason for stopping smoking). There were no differences between never and ever users of COCs in the reasons given for deciding to stop smoking. Neither were there any discernable differences between the percentage of women in each contraceptive group stopping in each calender year; annual percentage stopping varied between 1.5 and $6.8 \%$ per year, with no regular pattern (data not shown).

\section{Discussion}

Although the nested case-control study results were based on small numbers, they suggest that our previous risk estimates for myocardial infarction associated with COC use ${ }^{2}$ were not biased by changes in the smoking habits of COC users during the course of the Oral Contraception Study. The data were too sparse to allow us to examine the interaction between COC use and smoking. It is unlikely, however, that our previous finding of important interaction ${ }^{2}$ has been compromised. The RCGP findings, therefore, can still guide clinical practice.

On the other hand, our previous results for the risk of myocardial infarction associated with smoking are likely to have been underestimates. Comparison of the odds ratio obtained when using smoking at recruitment with that which used smoking at time of event, suggest a possible $40 \%$ underestimation of risk. This size of discrepancy could introduce bias if information collected at recruitment were used to assess within the cohort the effects of smoking, such as estimated effects of lifetime smoking on life expectancy. ${ }^{6}$

Comparison of the information supplied by the women retrospectively with that reported 


\section{KEY POINTS}

- Changes in smoking status have not biased our previous estimates of the risk of myocardial infarction associated with COC use.

- Fewer regular smokers who have used COCs stop smoking than non-users.

- Changes in smoking status would have underestimated the risk of myocardial infarction associated with smoking.

- When examining the lifetime effects of smoking within a cohort, changes in smoking must be allowed for.

by the general practitioner showed a good level of agreement. Approximately $13 \%$ of women said by their doctor to be smokers at recruitment were estimated from their questionnaire responses to have been non-smokers at this time. Nearly two thirds of this group denied ever smoking regularly. The remainder gave a starting date that was after recruitment or a stopping date before recruitment, and therefore would have been deemed to be nonsmokers at entry to the Oral Contraception Study. Part of these discrepancies are probably explained by the way we defined regular smoking in the questionnaire. Thus, a woman who had smoked for only a few months, or intermittently, at recruitment could have been correctly reported not being a regular smoker at this time. On the other hand, her general practitioner who was asked only to record number of cigarettes smoked on the day of recruitment would have classified her as a current smoker.

Compared with women said by their general practitioner to smoke heavily at recruitment (15 or more cigarettes daily), moderate smokers (daily consumption less than 15 cigarettes) were more likely to deny ever smoking regularly when asked later in life. Other studies have found that former light smokers may reclassify themselves as lifetime non-smokers. ${ }^{78}$ Conversely, a sizeable proportion (approximately $39 \%$ ) of doctor designated moderate smokers claimed to be heavier smokers on the questionnaire. Perhaps the women felt less able to reveal their true smoking habits to their doctor than in a self completed questionnaire. Alternatively, the women may have increased their cigarette consumption during the course of the study; the information about number of cigarettes smoked at recruitment was based on current smoking habits or those at time of stopping smoking.

Given the reasonable quality of the self reported smoking information, we also examined some characteristics associated with stopping smoking. Few studies have followed up a large cohort of women from the general population. ${ }^{9}{ }^{10}$ The Framingham Heart Study in the $\mathrm{USA}^{9}$ and a panel study of living conditions in Sweden, ${ }^{10}$ both found that older women, and those who smoked lightly, were more likely to stop smoking. Higher educa- tional attainment was also a positive predictor of stopping smoking.

In our study, there was a higher proportion of ex-smokers among nulliparous women at recruitment, possibly reflecting changes in smoking as a consequence of future pregnancies. It is noteworthy, however, that comparatively few women $(8 \%)$ gave this as the reason for stopping smoking. To our knowledge, this is the first study to report changes in smoking habits among a cohort of COC users. We have previously shown that users of COCs are more likely to smoke, and smoke more heavily, than women not using this method of contraception. ${ }^{4}$ We have now shown that COC users are less likely than non-users to stop smoking, even though there has been considerable publicity about the harmful effects of combining smoking with COC use. We thought that we might have seen peaks of stopping smoking in ever users during the years when there was particular publicity about these effects (such as 1977 and 1981 when the Oral Contraception Study had two major publications on this issue). ${ }^{11}$ It was disappointing that we could not detect such peaks, although many women will have stopped using COCs by the time these publications appeared and so might have felt that the results were irrelevant to them. None the less, health professionals need to continue to reinforce the message that COC users should not smoke.

Compared with women who responded to the questionnaire, the 1724 non-respondents were more likely to be smokers at recruitment ( $40.8 \%$ versus $53.7 \%$ respectively), of manual social class $(67.5 \%$ versus $78.3 \%)$ and of higher parity $(41.8 \%$ versus $49.6 \%$ had three or more children). There were no differences in terms of age, area of residence or use of COCs. The observed differences could change our conclusions if the pattern of changes in smoking habits among non-responding smokers was materially different to that of responding smokers. We are unable to determine if this is the case, although given the low proportion of non-responders to the questionnaire, any effects are probably small. Similarly, the small number of women who had to be excluded because of missing or incomplete smoking information suggests that any errors introduced by differences in changes in smoking habits between this group and those with complete information would probably be modest.

The Oral Contraception Study has been subject to major losses to follow up, mainly because the women moved from the practice area of the recruiting doctor or because the doctor withdrew from the study. Women no longer under observation tend to be younger, of higher social class, and lower parity than those still in the study. There are no differences in smoking habits at recruitment between those remaining under observation and those who do not. Recent analyses of mortality data have shown that the study's results have not been substantially biased by the large losses to follow up. ${ }^{12}$ It is unlikely, therefore, that loss to follow up could have biased the results presented here. 
Our results show that the changes in smoking habits experienced by the cohort have not compromised our previous COC related findings, but will have underestimated the effects of smoking. Using the updated smoking information, we are now in a position to be able to assess the lifetime effects of smoking within a large cohort of women living in Britain.

We thank the hundreds of doctors who have contributed to the Oral Contraception Study during the past 28 years, and the thousands of women who completed the questionnaire.

Funding: the postal questionnaire was funded by an unconditional grant from Wyeth-Ayerst International Inc. Additional support for the study has been received from the Royal College of General Practitioners, Schering AG, and Schering Health Care.

Conflicts of interest: none.

1 Royal College of General Practitioners' Oral Contraception Study. Mortality among oral contraceptive users. Lancet 1977;ii:727-31.

2 Croft P, Hannaford PC. Risk factors for acute myocardial infarction in women: evidence from the Royal College of General Practitioners' Oral Contraception Study. BMF 1989;298:165-8.
3 Hannaford PC, Croft PR, Kay CR. Oral contraception and stroke: evidence from the Royal College of General Practitioners' Oral Contraception Study. Stroke 1994;25:935-42.

4 Royal College of General Practitioners. Oral contraceptives and health. London: Pitman Medical, 1974.

5 Epidemiological graphics, estimation and testing package. Seattle: Statistics and Epidemiology Research Corporation, 1990.

6 Phillips AN, Wannamethee SG, Walker M, et al. Life expectancy in men who have never smoked and those who have smoked continuously: 15 year follow up of a large cohort of middle aged British men. BMF 1996;313:907-8.

7 Persson P, Norell SE. Retrospective versus original informaPersson P, Norell SE. Retrospective versus original informa-
tion on cigarette smoking: implications for epidemiological studies. Am F Epidemiol 1989;130:705-12.

8 Britten N. Validity of claims to lifelong non-smoking at age 36 in a longitudinal study. Int f Epidemiol 1988;17:525-9.

9 Freund KM, D'Agostino RB, Belanger AJ, et al. Predictors of smoking cessation: The Framingham Study. Am f Epidemiol 1992;135:957-64.

10 Tillgren P, Haglund BJA, Lundberg M, et al. The sociodemographic pattern of tobacco cessation in the 1980s: results from a panel study living condition surveys in Sweden. $\mathcal{F}$ Epidemiol Community Health 1996;50:625-30.

11 Royal College of General Practitioners' Oral Contraception Study. Further analyses of mortality in oral contraceptive users. Lancet 1981;i:541-6.

12 Beral V, Hermon C, Kay C, et al. Mortality in relation to method of follow-up in the Royal College of General Practitioners' Oral Contraception Study. In: Hannaford PC, Webb AMC, eds. Evidence-guided prescribing of the pill. London: Parthenon Publishing 1996:327-39. 